\title{
Medical Image Enhancement Optimizing Contourlet Transformation by using PSO Approach
}

\author{
Ritika Madaan \\ Dept. of Electronics \& Communication, \\ PIET, Kurukshetra University, \\ Kurukshetra
}

\author{
Satish Saini \\ Dept. of Electronics \& Communication, \\ PIET, Kurukshetra University, \\ Kurukshetra
}

\begin{abstract}
Image contrast enhancement is a feature for the enhancement of image. Images contrast is a feature which has been utilize for the proper visualization of image. The nuclei of the hydrogen atoms in the biological tissue adjusts themselves to the magnetic field, afterwards a radio-magnetic pulse will raise their energy level further, when the pulse ends they will relax and during the relaxation this energy will be transmitted from the atoms. The transmitted signal then be detected by the equipment and processed further into the pixels which turn up makingthe biomedical image. This work is more focused on medical images but the methods described here can be applied to any kind of image. The medical images have been enhanced by using the countourlet transformation. In this work the contourlet transform is flexible enough to also do the regular 2-D separable wavelet transform. In this paper counter let transformation is being used for enhancement of medical image. Then contourlet transformation is optimized by using PSO approach.
\end{abstract}

\section{Keywords}

Image enhancement, medicalimage, correlation, EPI and SSIM, PSO

\section{INTRODUCTION}

\subsection{Image Enhancement}

Image enhancementis enhancing the impression of data in pictures for human viewers and giving 'better' input for other computerized picture handling methods. The important goal of image enhancement is to change credits of a picture to make it more suitable for a given assignment and a particular spectator. During this procedure, one or more characteristics of the picture are adjusted. The decision of traits and the way they are altered are particular to a given work. There exist numerous procedures that can improve an advanced picture without ruining it. The enhancement methods can broadly be divided in to the following two categories:

1. Spatial Domain Methods

2. Frequency Domain Methods

1.1 Spatial Domain Method:The estimation of a pixel with directions $(\mathrm{x}, \mathrm{y})$ in the improved picture is the consequence of performing some operation on the pixels in the area of $(x, y)$ in the data picture, F. Neighborhoods can be any shape, yet for the most part they are rectangular.

1.2 Frequency Domain Method:Picture upgrade in the recurrence space is direct. Author just process the Fourier change of the picture to be upgraded, duplicate the outcome by a channel (instead of convolve in the spatial space), and take the opposite change to create the improved picture. The thought of reducing so as to obscure a pictureits high recurrence parts, or honing a picture by expanding the size of its high recurrence segments is naturally straightforward. Understanding recurrence space ideas is imperative, and prompts upgrade methods that won't have been considered by limiting thoughtfulness regarding the spatial area.

\subsection{ImageEnhancement Techniques}

\subsubsection{Adaptive Median Filter}

The principle thought of the middle channel is to gone through the picture pixel by pixel, supplanting every pixel with the middle of neighboring pixels. The example of neighbors is known as the "window", which slides, pixel by pixel, over the whole flag. The middle channel is a nonlinear channel which, under specific conditions, can safeguard edges, and it is frequently used to evacuate clamor as a prehandling venture to enhance the aftereffects of later preparing (for illustration, growth division).

\subsubsection{Histogram Equalization}

When an image goes through this technique, this adjusts the contrast of the image using the image's histogram. The technique typically raises the global contrast of images, especially when the data of the image is laid-out by close contrast values. This permits for areas of depressed local contrast to gain a higher contrast. Histogram equalization carries out this by efficaciously distributing the most frequent intensity values. The method is effectual in images with backgrounds and foregrounds that are both bright or both dark and this is in particular correct for the mammograms

\subsubsection{Histogram Modified Local contrast Enhancement}

Reliably scatters the yield histogram by using cumulated histogram as its mapping limit. In any case it makes over change in the yield picture which prompts loss of more neighborhood information in the primary mammogram. One more issue with $\mathrm{HE}$ is its incomprehensible retrogressive differentiation advantages of mapping limits and the manysided quality overhaul potential should be progressed without the fine unobtrusive components in the mammogram picture. Remembering the deciding objective to reduce the level of overhaul that possible got by HIM, the information histogram can be changed so the adjusted histogram is more like a reliably spread histogram. HM-LCE framework merges a two stage taking care of both histogram adjustment and neighborhood contrast change system. The standard focus of this procedure is to find a changed histogram that is closer to uniform histogram and to have the impact amidst modified and information histogram little, which in this way extends the likelihood of picture contrast overhaul and resultant picture would be the more imperative to the data picture. 


\subsection{Contrast limited adaptive histogram equalization (CLAHE)}

CLAHE is a different strategy than simple AHE as in Contrast Limiting. Adaptive equalization can over amplify the noise and so CLAHE is developed so that over amplification could be prevented by limiting the contrast. It differs from standard histogram leveling in the admiration that the versatile technique considers a few histograms, each relating to a different segment of the picture, and employs them to redistribute the daintiness estimations of the image. This is how it is suitable for enhancing the neighborhood difference of mammogram pictures and bringing out more detailed information to the contemplation of specialists.

\subsection{Fuzzy contrast enhancement}

Fluffy picture improvement is in light of dull level mapping in to a feathery plane, using an interest change limit. The fact of the matter is to deliver a photo of higher distinction than the primary picture by giving a greater weight to the dim levels that are closer to the mean faint level of the photo than to those that are more inaccessible from the mean. An intriguing work which uses the same thought is executed for redesigning the distinction of mammograms, yet disastrously as a result of compelled time, the figuring shown in the main work, to the best understanding, is lacking with respect to a couple purposes of hobby and the overhauled picture coming to fruition as a result of its use is obviously wrong for a plan of pixels. Therefore for whatever is left of this report, further elucidation of the computation is skipped and grown all the more on the outwardly weakened de-convolution and the last test outcomes.

\section{APPROACHES USED}

\subsection{Counter let transformation}

The Contourlet transform makes use of a double filter bank schemeto get the smoothened contours of images. In this twofold filter bank, the Laplacian pyramid (LP) is initially applied to catch the point discontinuities, and afterward a directional Filter bank (DFB) is utilized so as to shape those point discontinuities into straight structures. The Laplacian pyramid (LP) disintegration just delivers one band-pass picture in a multidimensional sign handling that can stay away from recurrence scrambling. Also, directional Filter bank (DFB) is fit for high recurrence since it will release the low recurrence of signs in its directional sub-groups. This is the motivation to consolidate DFB with LP, which is multi-scale disintegration and evacuate the low recurrence. The Contourlet change is propelled by the human visual framework and Curve let change which can catch the smoothness of the form of pictures with various extended shapes and in assortment of headings. Be that as it may, it is hard to inspecting on a rectangular matrix for Curve let change since Curve let change was produced in consistent space and bearings other than level and vertical are altogether different on rectangular framework. Accordingly, the Contourlet change was proposed at first as a directional multidetermination change in the discrete area.

\subsection{Particle swarm optimization}

Particle swarm optimization (PSO) is a computing technique which optimizes a problem by using a series of iterations attemptingto mend a candidate solution with regard to a given measure of quality's optimizes a problem by having a population of candidate solutions, here named as dubbed particles, and making these particles move around in the search-space according to a simple mathematical formulae over the particle's position and velocity. Each particle's movement is regulated by its local best position and is also guided toward the best positions in the search-space, which are updated as better positions found by other particles.PSO makes few or no assumptions about the problem being optimized and can search very large spaces of candidate solutions.

\section{RELATED WORK}

Abishek Gupta et al [1] "Evolutionary Approach and Spline Interpolation for Contrast Enhancement" In this paper, image contrast enhancement strategies are explored and two novel approaches are proposed. The important philosophy uses a Super Resolution based Convolution bit in Lab shading space which is enhanced using Genetic Calculations, for the transformative period of the piece values. The used health limit tends to give a concordance in the middle of distinction and normal for pictures. The second approach is called Spline Interpolation which meets desires by fitting a relentless three dimensional curve through $(0,0$, and 0$)$, the present pixel's RGB regard and $(255,255,255)$.

Peng Fengbet al [2] "Enhancing retinal image by the Contourlet transform" The evaluation of retinal pictures is broadly used to offer specialists some assistance with diagnosing numerous infections, for example, diabetes or hypertension. Because of the securing process, retinal pictures regularly have low dark level differentiation and element range. This issue might genuinely influence the indicative strategy and its outcomes.Hereanothermultiscalesystemisdisplayedforretinalpicturecontrast improvement taking into account the Contourlet change. The Contourlet change has preferred execution in speaking to edges over wavelets for its anisotropy and directionality, and is in this way appropriate for multi-scale edge improvement. Creator change the Contourlet coefficients in relating sub groups by means of a nonlinear capacity and consider the commotion for more exact reproduction and better perception.

Alejandro F. Frangiet al [3] "Multiscale vessel enhancement filtering" The multiscale second request nearby structure of a picture (Hessian) is analyzed with the motivation behind building up a vessel enhancement filter. A vesselness measure is gotten on the premise of all eigen estimations of the Hessian. This measure is tried on two dimensional DSA and three dimensional aortoiliac and cerebral MRA information. Its clinical utility is appeared by the concurrent clamor and foundation concealment and vessel improvement in most extreme force projections and volumetric showcases.

MuneerAhmadet al [4] "An Integrated Approach for Medical Image Enhancement using Wavelet Transforms and Image Filtering" Medical image enhancement is a central advancement issue in software engineering. Upgraded pictures can give better knowledge of patient's illness and solution for cure. Specialists have been paying extraordinary thought over this testing issue yet a huge ideal arrangement is need of time. This paper shows a coordinated methodology for Medical image enhancement utilizing wavelet changes and picture separating. The objective smoothed clamor smothered picture beat for a critical rate of change by bringing noteworthy results over traditional image enhancement procedures.

Alex F. de Araujoet al [5] "New artificial life model for image enhancement" In this work, a strategy to enhance pictures in light of another counterfeit life model is introduced. The model is inspired on the conduct of a herbivore living being, the point at which this life form is in a 
specific domain and chooses its nourishment. This life form goes through the picture iteratively, selecting the more suitable sustenance and eating parts of it in every emphasis the way that the living being goes through in the picture is characterized by from the earlier learning about nature and how it ought to move in it. Here, the team displayed the control and discernment focuses of the life form, and additionally the recreation of its activities and consequences for nature. To exhibit the effectiveness of used technique quantitative and subjective consequences of the improvement of engineered and genuine pictures with low difference and diverse levels of noise are presented.

\section{PROBLEM FORMULATION}

Image contrast enhancement is a feature for the enhancement of image. Images contrast is a feature which has been utilize for the proper visualization of image. In these feature intensity value available in the image, plays a vital role for the purpose of visualization. Bio-medical images like x-ray, ultrasound etc. has drawback of low contrast constraints. Due to the low level of contrast, image feature cannot be proper visualized. Various approaches have been proposed for the enhancement of bio-medical images. The selection of the signal resolution is the next key problem came across in the digital processing of both 1-D and 2-D signals. This specifies the sampling period in the case of time series or the pixel size in the case of images. Altering the resolution of an image allows both global and detailed views of specific 1-D or 2-D signal components. The medical images have been enhanced by using the contour let transformation. The contourlet transform is flexible enough to also do the regular 2-D separable wavelet transform. This transformation use different decomposition filters and reconstruction filters for the process of image enhancement. The image enhancement process has been optimized by using different approaches that has been used for evolution on the basis of fitness function.

\section{METHODOLOGY}

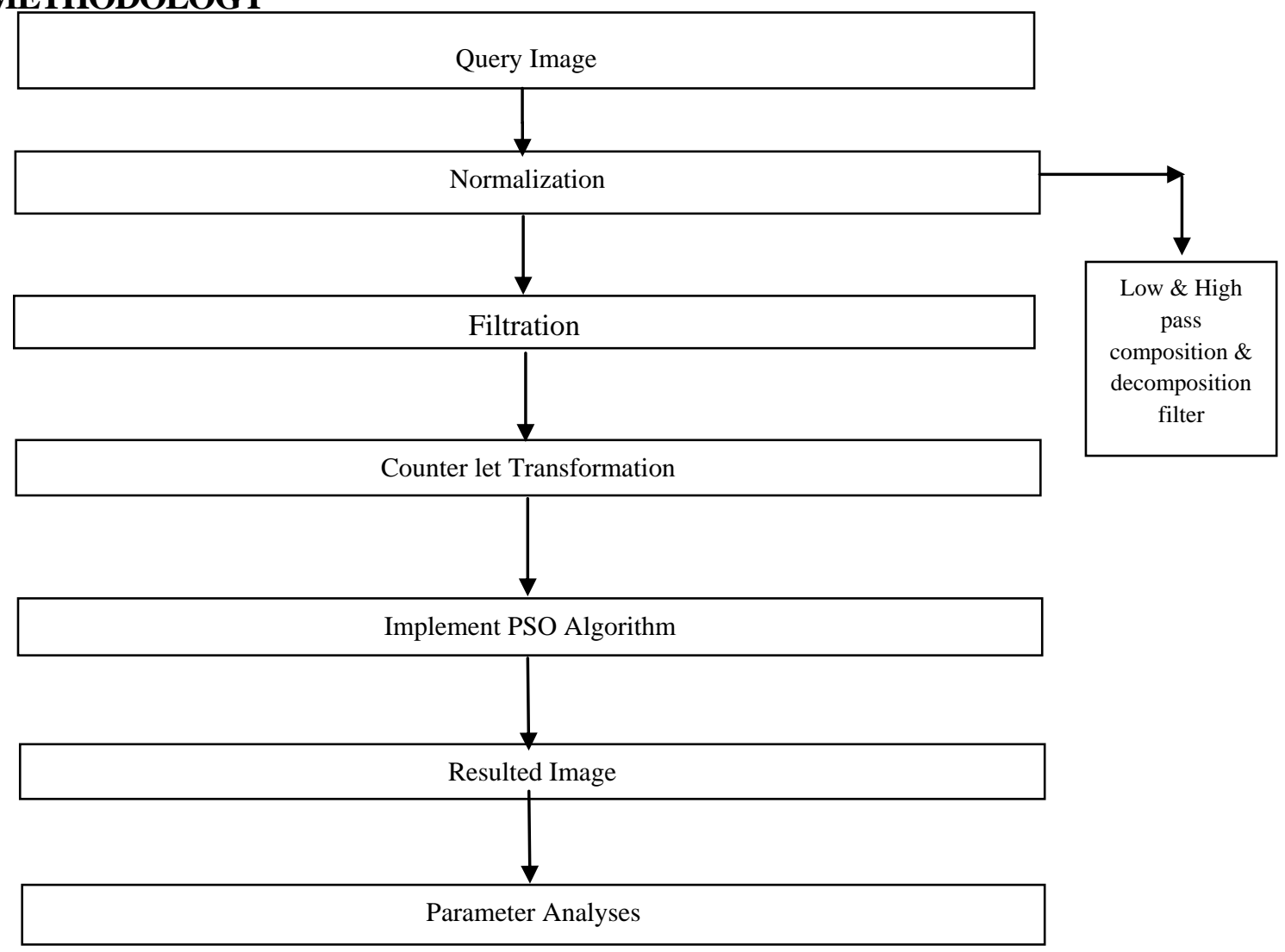

Fig: Flow of Work 


\section{RESULTS AND DISCUSSIONS}

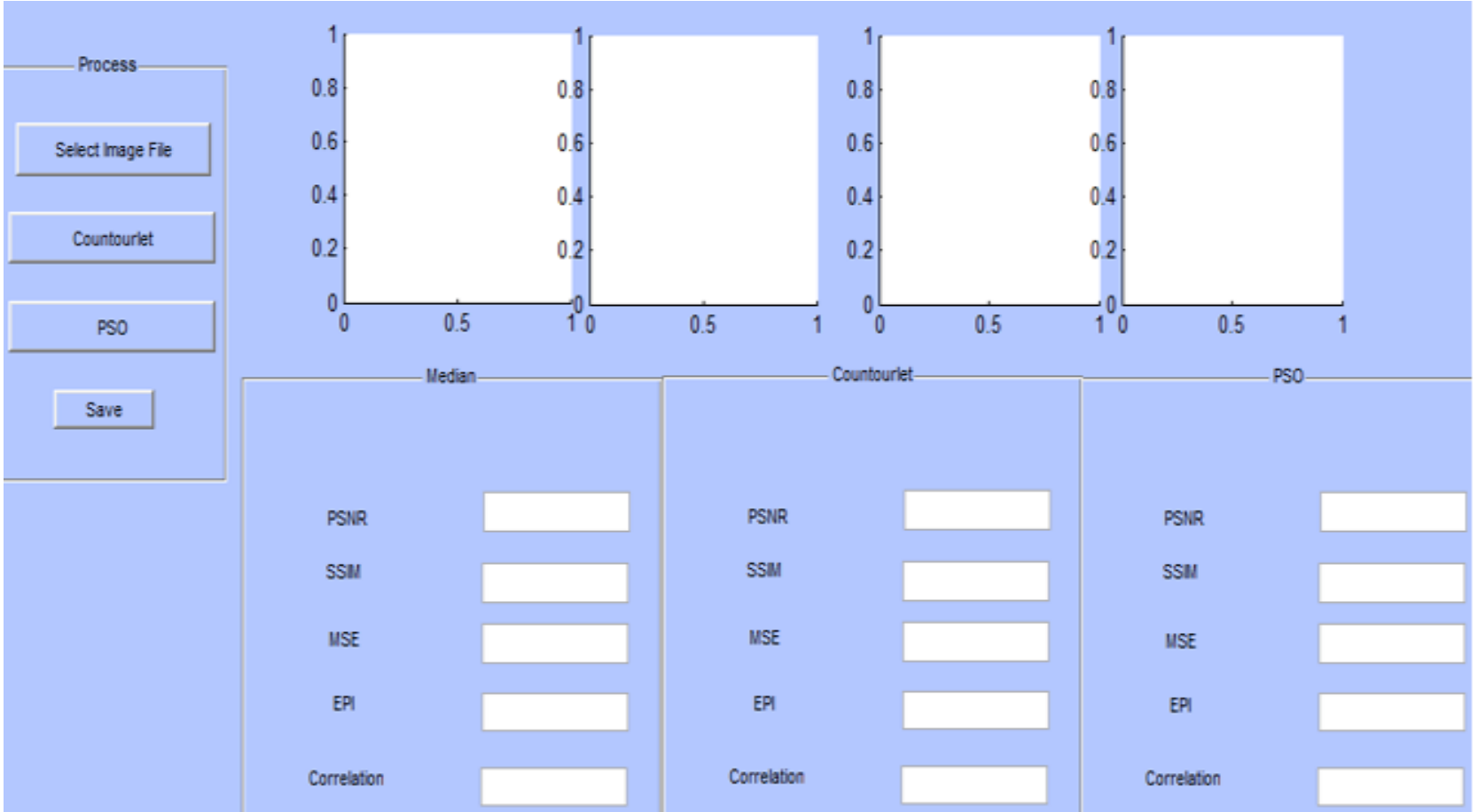

Fig 1: Graphic user interface for the proposed work

This figure corresponds to the graphic user interface that has been projected for various operations that have to be performed on the basis of different computational steps. This user interface consists of different buttons and edits boxes, axes to represent the image, the buttons are used for these axes have been used performing variousoperations that has been assignedbehindtheirretrieval.

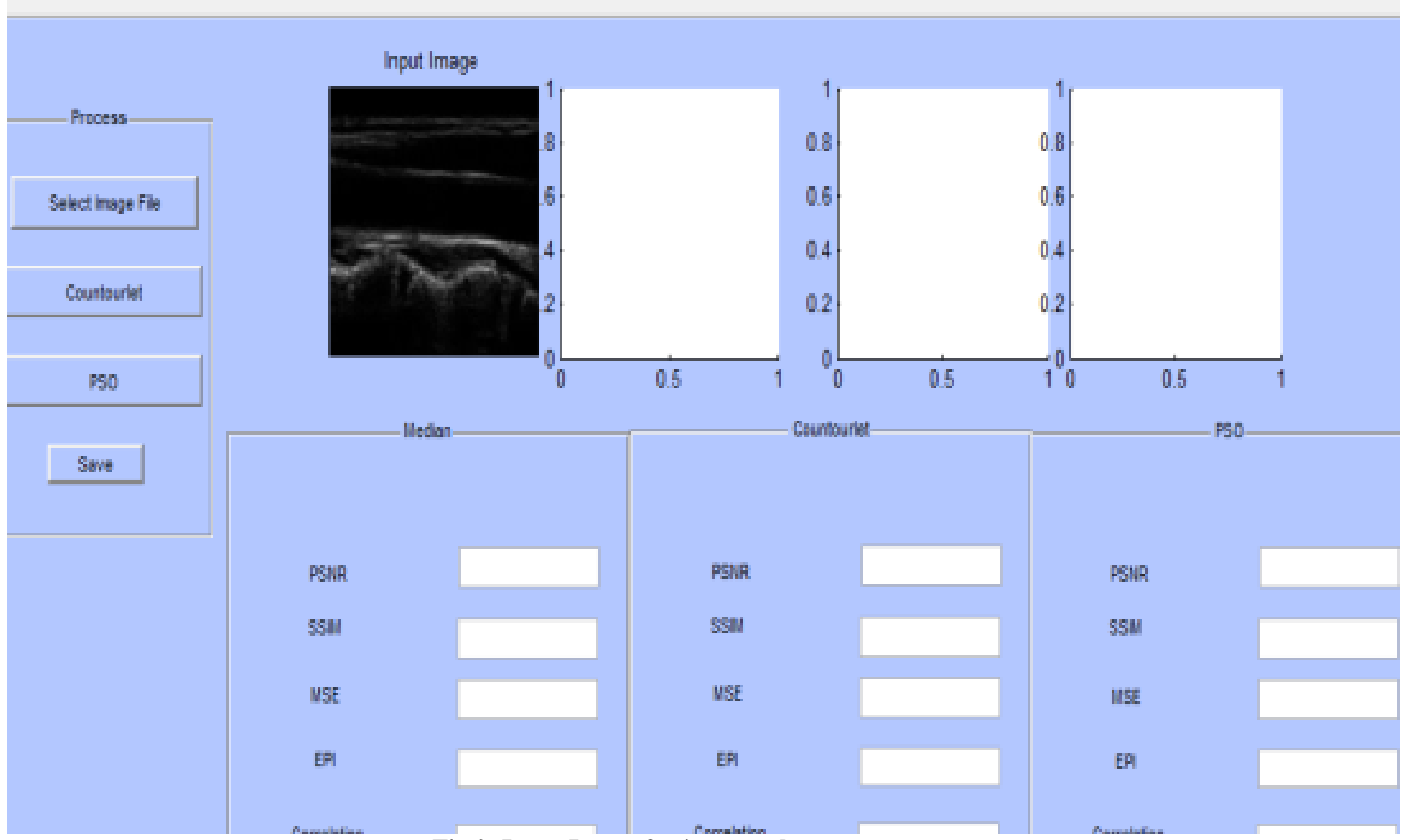

Fig 2: Input Image for image enhancement 
This figure represents the input image that has been chosen as a sample for image enhancement. The input image featurescannot be easily seen bared eyes due to the blurriness.
Different noise intensities are there in the input image. To enhance the image these intensities needed to be removed.

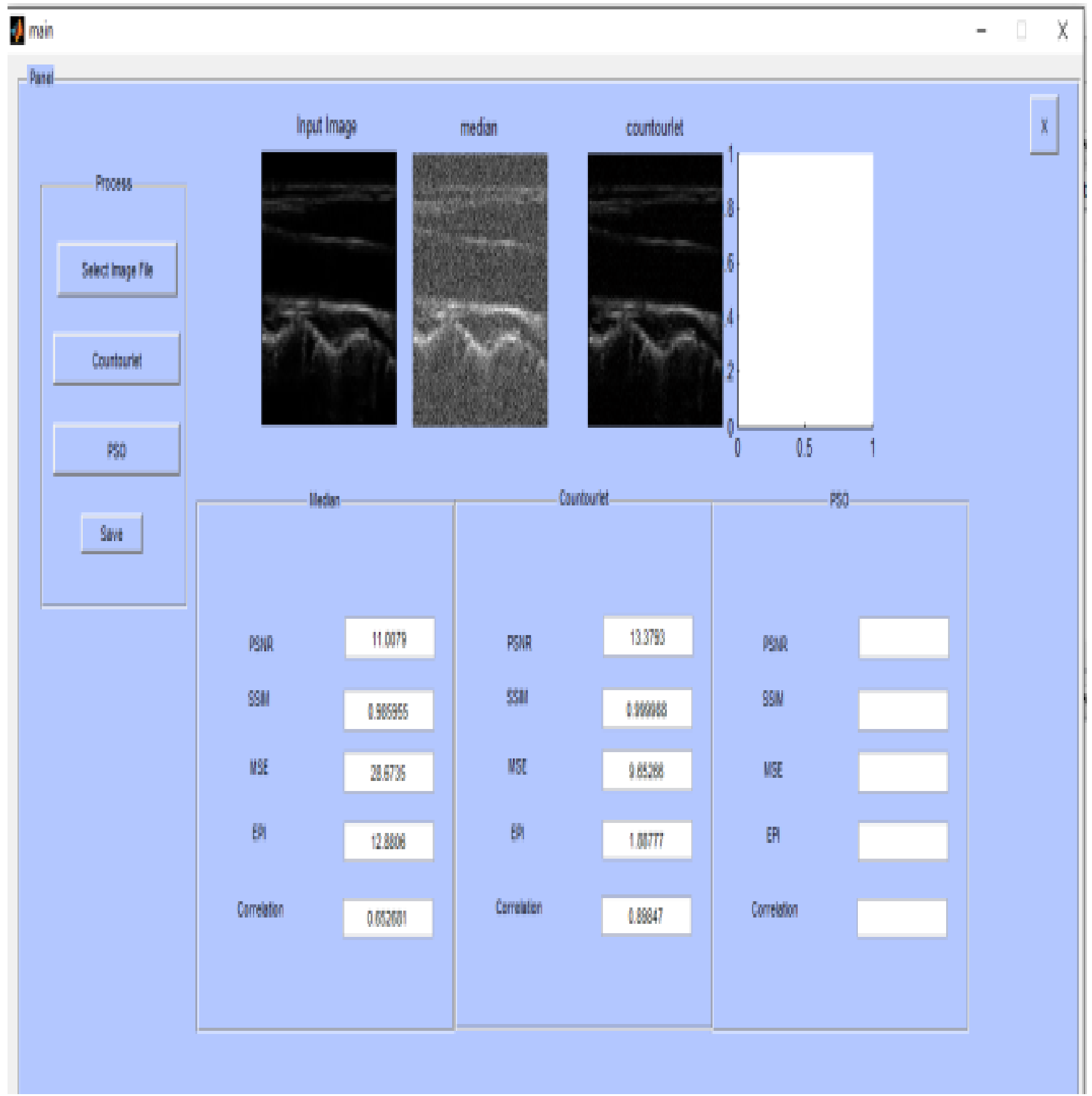

Fig 3: Contourlet transformation on the input image for image enhancement

This figure describes that the image that has been touched up by using contourlet transformation. This operation use different decomposition filters that divide the image into different segments. The noise removal filter eliminates the noise from the image and then reconstruction filters are implemented to reconstruct the image. 


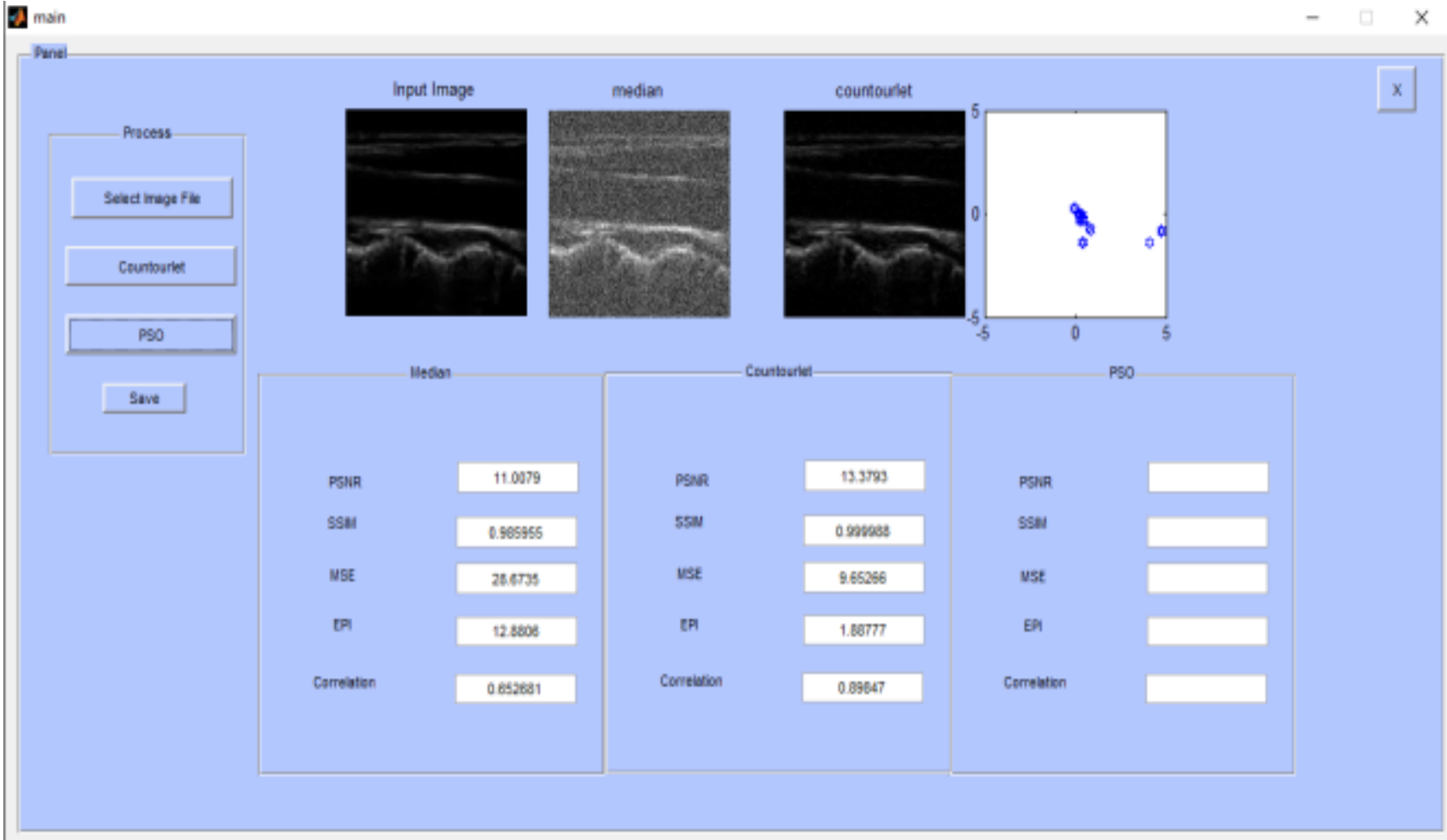

\section{Fig 4: PSO optimization for image enhancement}

This figure depicts the process where blue dots represent population of random solutions. The image that has been enhanced by using the PSO algorithm can be seen on the completion of the PSO algorithm.Also theparameters are defined for PSO.

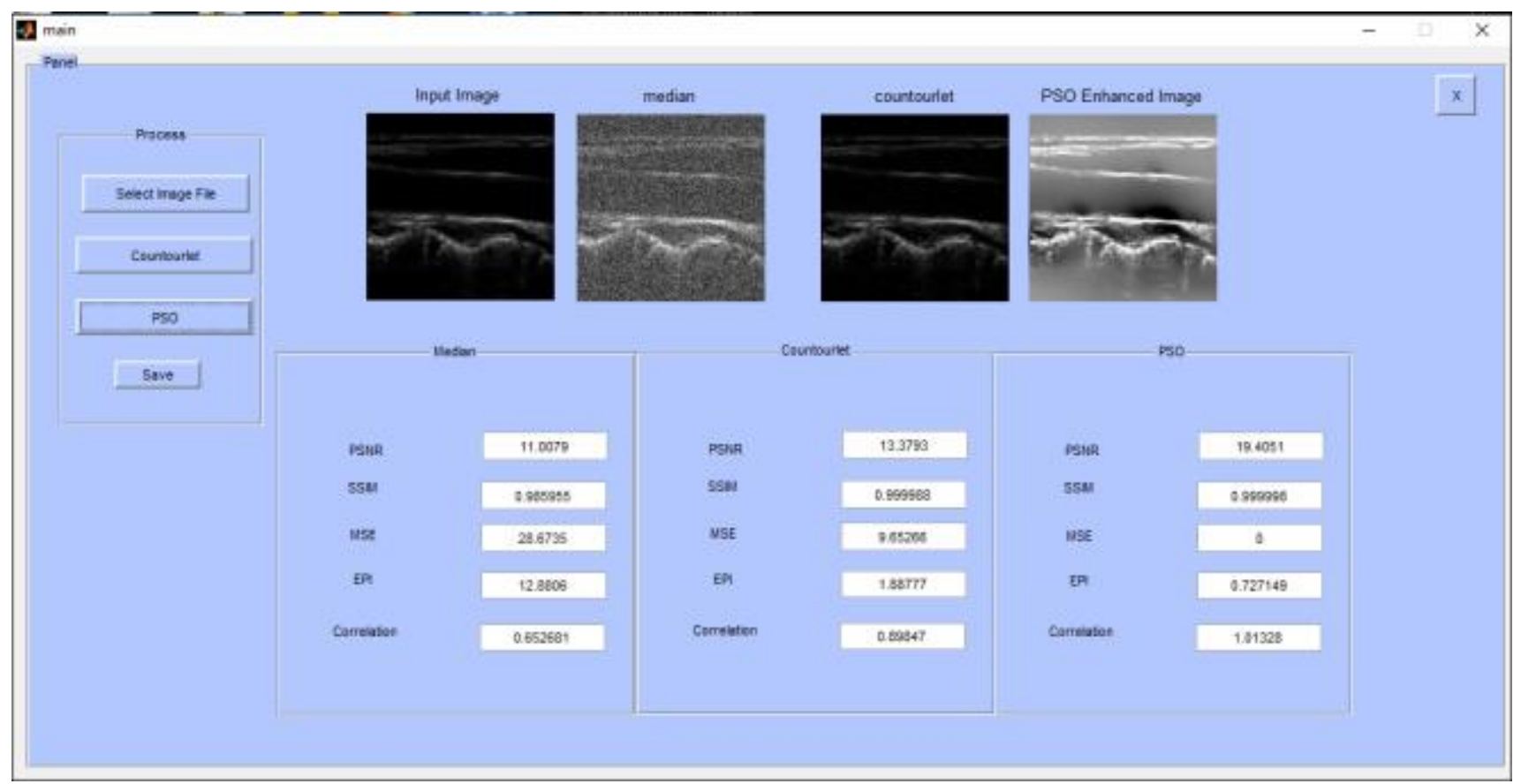

Fig 5: Calculation of different parameters

This figure represents the evaluation of various parameters for comparison of the purposed work with previous approaches. In this calculations of different parameters like
PSNR,MSE,SSIM,EPI and Co-relation have been measured for performance computation of image by using contour-let transformation and PSO algorithm. 
Table 1: Comparison Table for performance evaluation

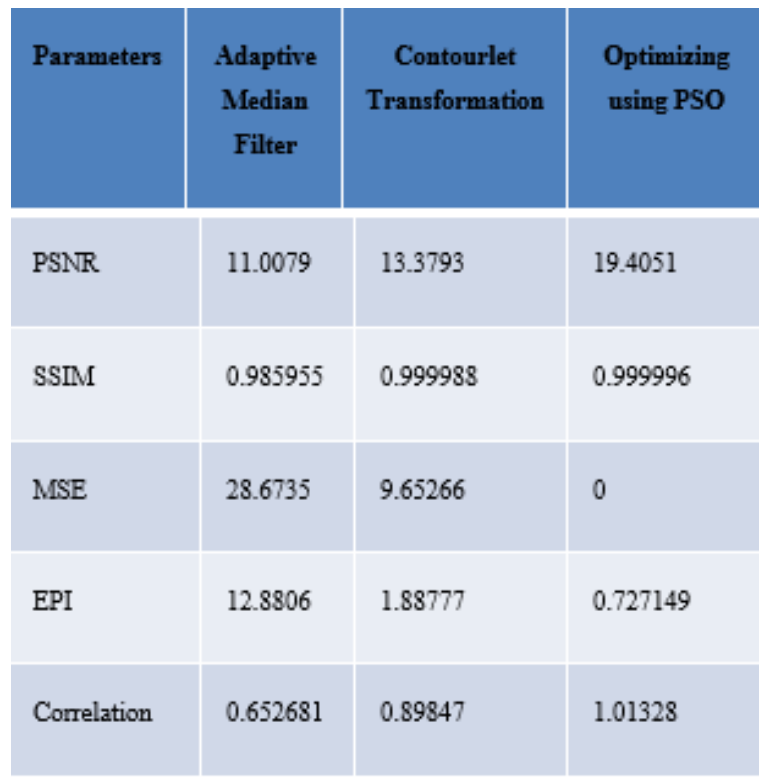

\section{CONCLUSION}

Image enhancement is the process for image quality improvements by using different operators. In the process of image enhancement different approaches have been used for image enhancement. In the purposed work the contourlet transformation has been used for the process of image enhancement. The contourlet apply LP and FB decomposition filter so that the image can be divided into different segments.

After carrying out the contourlet transformation the PSO algorithm is implemented to optimize the output from contourlet. This approach is based on the natural social behavior of bird flocking. After these steps various parameters have been analyzed for validation of purposed work. On the basis of different parameters that are PSNR, MSE, SSIM and EPI one can conclude that the image enhancement with hybridization of contourlet and PSO provides better performance thanotherapproaches.

\section{FUTURE SCOPE}

In future the work can be done on Speckle noise. One can use classification Method which is use to find the area for classification by using region of instruct.

\section{REFERENCES}

[1] http://users.dcc.uchile.cl/ jsaavedr/libros/dip_gw.pdf

[2] Abishek Gupta, Ajesh Nag, JatenSavani “Evolutionary Approach and Spline Interpolation for Contrast Enhancement" IEEE Second International Conference on Image Information Processing, pp. 181-186, 2013.

[3] Peng Feng "Enhancing retinal image by the Contourlet transform", IEEE Conf. on Counterlet transform, 2007, pp 516-522.

[4] Alejandro F. Frangi "Multiscale vessel enhancement filtering", IEEE Conf. on enhancement, 1998, pp 130137.

[5] Muneer Ahmad "An Integrated Approach for Medical Image Enhancement using Wavelet Transforms and Image Filtering", IEEE Conf. on Life Science Journal, 2014, pp 6-11.

[6] Alex F. de Araujo "New artificial life model for image enhancement", IEEE Conf. on image enhancement, 2013, pp 6-16.

[7] Swagatam Das1, "Bacterial Foraging Optimization Algorithm: Theoretical Foundations, Analysis, and Applications", IEEE Conf. on BFOA, 2014, PP 31-40.

[8] Nik ShahidahAfifi BT MdTaujuddin "Enhancement of Medical Image Compression by using Threshold Predicting Wavelet-Based Algorithm", IEEE Conf. on Medical Imahges, 2011, pp 43-52.

[9] Janani, V; Dinakaran, M. "Infrared image enhancement techniques- A review", in Current Trends in Engineering and Technology (ICCTET), 2014, ISBN 978-1-47997986-8, pp 167-173.

[10] Bhattacharya, S; Gupta, S.; Subramanian, V.K. "Localized image enhancement", 2014, pp 1-6. 